J. Korean Math. Soc. 51 (2014), No. 4, pp. 655-663

http://dx.doi.org/10.4134/JKMS.2014.51.4.655

\title{
RINGS WITH A FINITE NUMBER OF ORBITS UNDER THE REGULAR ACTION
}

\author{
Juncheol Han and SANGWon Park
}

\begin{abstract}
Let $R$ be a ring with identity, $X(R)$ the set of all nonzero, non-units of $R$ and $G(R)$ the group of all units of $R$. We show that for a matrix ring $M_{n}(D), n \geq 2$, if $a, b$ are singular matrices of the same rank, then $\left|o_{\ell}(a)\right|=\left|o_{\ell}(b)\right|$, where $o_{\ell}(a)$ and $o_{\ell}(b)$ are the orbits of $a$ and $b$, respectively, under the left regular action. We also show that for a semisimple Artinian ring $R$ such that $X(R) \neq \emptyset, R \cong \oplus_{i=1}^{m} M_{n_{i}}\left(D_{i}\right)$, with $D_{i}$ infinite division rings of the same cardinalities or $R$ is isomorphic to the ring of $2 \times 2$ matrices over a finite field if and only if $\left|o_{\ell}(x)\right|=\left|o_{\ell}(y)\right|$ for all $x, y \in X(R)$
\end{abstract}

\section{Introduction and basic definitions}

Throughout this paper all rings are assumed to be rings with identity $1 \neq$ 0 . Let $R$ be a ring, $X(R)$ (simply, denoted by $X$ ) the set of all nonzero nonunits of $R, G(R)$ (simply, denoted by $G$ ) the group of all units of $R$, and $J$ the Jacobson radical of $R$. In this paper, we consider two group actions of $G$ on $X$ given by $((g, x) \longrightarrow g x)$ (resp. $\left.\left((g, x) \longrightarrow x g^{-1}\right)\right)$ from $G \times X$ to $X$ called the left (resp. right) regular action. For each $x \in X$, recall that the orbit of $x$ by $o_{\ell}(x)=\{g x: \forall g \in G\}$ (resp. $o_{r}(x)=\left\{x g^{-1}: \forall g \in G\right\}$ ) under the left (resp. right) regular action of $G$ on $X$. Also recall that the stabilizer of $x$ by $\operatorname{stab}_{\ell}(x)=\{g \in G: g x=x\}$ (resp. $\operatorname{stab}_{r}(x)=\{g \in G: x g=x\}$ ) under the left (resp. right) regular action of $G$ on $X$. $G$ is said to be transitive on $X$ (or $G$ acts transitively on $X$ ) under the left (resp. right) regular action if there is an $x \in X$ with $o_{\ell}(x)=X$ (resp. $o_{r}(x)=X$ ). In [1, Lemma 2.6, Theorem 2.7], Cohen and Koh have shown that (1) if $R$ is the ring of $2 \times 2$ matrices over a finite field $F$, then $\left|o_{\ell}(x)\right|=\left|o_{\ell}(y)\right|=|F|^{2}-1$ for all $x, y \in X$; (2) if $R$ is a semisimple ring, then there exists a compact ring topology on $R$ and $\left|o_{\ell}(x)\right|=\left|o_{\ell}(y)\right|$ for all $x$ and $y$ in $X$ if and only if $R$ is a finite field, $R$ is $F \times F$, where $F$ is a finite field with more than two elements or $R$ is isomorphic to the ring of $2 \times 2$ matrices over a finite field. Let $M_{n}(R)$ be a matrix ring of

Received July 2, 2012; Revised March 17, 2014.

2010 Mathematics Subject Classification. Primary 16W22; Secondary 16P20.

Key words and phrases. left (right) regular action, orbit, left Artinian ring.

This study was supported by research funds from Dong-A University. 
$n \times n$ matrices over a ring $R$. In Section 2, we will show that (1) for a ring $R=M_{n}(F)$ with $n \geq 2$, where $F$ is a finite field, if for some $a \in X$, we have $\operatorname{rank}(a)=s$, then $\left|o_{\ell}(a)\right|=\left|o_{r}(a)\right|=\left(|F|^{n}-1\right)\left(|F|^{n}-|F|\right) \cdots\left(|F|^{n}-|F|^{s-1}\right)$; (2) if $R$ is a semisimple Artinian ring such that $X \neq \emptyset$, then $R \cong \oplus_{i=1}^{m} M_{n_{i}}\left(D_{i}\right)$, with $D_{i}$ infinite division rings of the same cardinalities or $R$ is isomorphic to the ring of $2 \times 2$ matrices over a finite field if and only if $\left|o_{\ell}(x)\right|=\left|o_{\ell}(y)\right|$ for all $x, y \in X(R)$.

In [2], the rings with a finitely many orbits under the left regular action on $X$ by $G$ are considered and some properties are obtained. By Hirano [3], a full characterization of these rings was given, in that they are precisely rings that are a product of a finite ring and finitely many left Artinian left uniserial rings. In Section 3, we also investigate rings with a finitely many orbits on the left and show that the structures of these rings are determined according to some conditions of their Jacobson radicals. In [1, Lemma 2.2], Cohen and Koh have also shown that if $R$ is a ring such that $X \neq \emptyset$, then $G$ is transitive on $X$ under the left regular action if and only if $R$ is a local ring such that $J^{2}=0$ and $J$ is a one-dimensional vector space over $R / J$. We prove that $(1)$ If there exist two orbits under the left (resp. right) regular action on $X$ by $G$, then $R$ is a local ring, or $R$ is isomorphic to a direct product of two division rings; (2) $R$ is a ring in which there exist three orbits under the left (right) regular action on $X$ by $G$ if and only if $R$ is isomorphic to $M_{2}\left(\mathbb{Z}_{2}\right)$. We also prove that if $R$ is a left Artinian ring in which $G$ is a cyclic group, then $R$ is a finite ring, and also for a ring $R$ with a finite union of orbits under the left (right) regular action, then $R$ is a finite ring.

\section{Regular action in a matrix ring}

In [1, Lemma 2.6], it was shown that if $R$ is the ring of $2 \times 2$ matrices over a finite field $F$, then $\left|o_{\ell}(x)\right|=\left|o_{\ell}(y)\right|=|F|^{2}-1$ for all $x, y \in X$. In this section, we will generalize the result by considering the ring of $n \times n$ matrices over a division ring $D$ (denoted by $M_{n}(D)$ ) for any positive integer $n \geq 2$. Observe that for some $a, b \in X, o_{\ell}(a)=o_{\ell}(b)$ if and only if $a$ is row equivalent to $b$.

Remark 1. Let $D$ be a division ring and $R=M_{n}(D)$ for any positive integer $n \geq 2$. Then we note that there are at least $|D|+1$ orbits in $R$ under the left regular action on $X$ by $G$. Indeed, for any $d \in D$, let $d e_{i j}$ denote the matrix in $R$ with $d$ in the $(i, j)$-position and 0 elsewhere. Let $a, b \in D(a \neq b)$ be arbitrary. Then $x_{a}=e_{11}+a e_{12}$ is not row-equivalent to $x_{b}=e_{11}+b e_{12} \in X$, which is equivalent to $o_{\ell}\left(x_{a}\right) \neq o_{\ell}\left(x_{b}\right)$ by Lemma 2.1. In addition, $e_{1,2}$ is not row equivalent to $x_{a}$ for any $a \in D$. Hence there are at least $|D|+1$ orbits in $R$ under the left regular action on $X$ by $G$. We can also note that $D$ is a finite field if and only if there is a finite number of orbits under the left (right) regular action on $X$ by $G$. Similarly, we also note that there are at least $|D|+1$ orbits in $R$ under the right regular action on $X$ by $G$. 
Lemma 2.1. Let $D$ be a division ring and $R=M_{n}(D), n \geq 2$. If for some $a, b \in X \operatorname{rank}(a)=\operatorname{rank}(b)$, then $\left|o_{\ell}(a)\right|=\left|o_{\ell}(b)\right|$.

Proof. Since $\operatorname{rank}(a)=\operatorname{rank}(b)$, then $b=U a V$ for some invertible matrices $U, V \in R$. Then $o_{\ell}(b)=o_{\ell}(U a V)=o_{\ell}(a V)$, and $X \leftrightarrow X V$ provides a bijection between $o_{\ell}(a V)$ and $o_{\ell}(a)$. Thus $\left|o_{\ell}(a)\right|=\left|o_{\ell}(b)\right|$.

Theorem 2.2. Let $F$ be a field and $R=M_{n}(F), n \geq 2$. Then we have the following:

(1) $\left|o_{\ell}(a)\right|=\left|o_{r}(a)\right|$ for all $a \in X$.

(2) The number of all orbits under the left regular action on $X$ by $G$ is equal to that of all orbits under the right regular action on $X$ by $G$.

Proof. (1) Note that $o_{\ell}\left(a^{t}\right)=o_{r}(a)^{t}$ for all $a \in X$, where $a^{t}$ is the transpose of $a$ and $o_{r}(a)^{t}=\left\{b^{t}: b \in o_{r}(a)\right\}$. Since $\operatorname{rank}(a)=\operatorname{rank}\left(a^{t}\right)$ by [4, page 227, Exercise 15], $\left|o_{\ell}(a)\right|=\left|o_{\ell}\left(a^{t}\right)\right|$ by Lemma 2.1. Clearly, $\left|o_{r}(a)\right|=\left|o_{r}(a)^{t}\right|$. Hence we have $\left|o_{\ell}(a)\right|=\left|o_{r}(a)\right|$ for all $a \in X$.

(2) Let $S$ be the set of all orbits under the left regular action on $X$ by $G$ (resp. $T$ be the set of all orbits under the right regular action on $X$ by $G$ ). Consider a map $\theta: S \rightarrow T$ defined by $\theta\left(o_{\ell}(a)\right)=o_{r}\left(a^{t}\right)$ for all $a \in X$. It is clear to show that $\theta$ is well-defined and one to one. Since $\theta\left(o_{\ell}\left(a^{t}\right)\right)=o_{r}(a), \theta$ is onto. Hence $\theta$ is bijective, and so $|S|=|T|$.

Theorem 2.3. Let $F$ be a finite field and $R=M_{n}(F), n \geq 2$. If $\operatorname{rank}(a)=s$ for some $a \in X$, then $\left|o_{\ell}(a)\right|=\left(|F|^{n}-1\right)\left(|F|^{n}-|F|\right) \cdots\left(|\bar{F}|^{n}-|F|^{s-1}\right)$.

Proof. Let $a_{1}=e_{11}+\cdots+e_{k k}+\cdots+e_{s s}$. Since $\operatorname{rank}(a)=\operatorname{rank}\left(a_{1}\right)=s$, $\left|o_{\ell}(a)\right|=\left|o_{\ell}\left(a_{1}\right)\right|$ by Lemma 2.1. Then $g a_{1}=\left[\overrightarrow{g_{1}}, \overrightarrow{g_{2}}, \ldots, \overrightarrow{g_{s}}, \overrightarrow{0}, \ldots, \overrightarrow{0}\right]$ for all $g \in G$, where $\overrightarrow{g_{k}}$ is the $k$-th column of $g(1 \leq k \leq s)$. Thus the number of elements in the orbit $o_{\ell}(a)$ equals the number of $s$-plets of linearly independent vectors $\left\{\overrightarrow{g_{1}}, \overrightarrow{g_{2}}, \ldots, \overrightarrow{g_{s}}\right\}$, and thus it equals $\left(|F|^{n}-1\right)\left(|F|^{n}-|F|\right) \cdots\left(|F|^{n}-\right.$ $\left.|F|^{s-1}\right)$.

Corollary 2.4. Let $F$ be a finite field and $R=M_{2}(F), n \geq 2$. Then $\left|o_{\ell}(a)\right|=$ $|F|^{2}-1$ for any $a \in X$.

Proof. Note that $\operatorname{rank}(a)=1$ for any $a \in X$. Hence $\left|o_{\ell}(a)\right|=|F|^{2}-1$ for any $a \in X$ by Theorem 2.3 .

Remark 2. For $R=M_{n}(F)$ ( $F$ is a finite field), $n \geq 2$, we note that the number of orbits of $a \in X$ with $\operatorname{rank}(a)=k$, which is equal to the number of row reduced echelon matrices, is easily seen (and well known) to be equal to the number of subspaces of dimension $k$ of $F^{n}$. In fact, the number subspaces of dimension $k$ of $F^{n}$ is $\left(\begin{array}{c}n \\ k\end{array}\right)_{q}=\frac{[n] !_{q}}{[k] !_{q}[n-k] !_{q}}$ for $q=|F|$, where $[n] !_{q}=(1)_{q}(2)_{q} \cdots(n)_{q}$ and $(i)_{q}=q^{i}-1$. 
Example 1. Let $R=\operatorname{Mat}_{2}\left(\mathbb{Z}_{2}\right)$, where $\mathbb{Z}_{2}$ is the Galois field of order 2 . Put $x=\left(\begin{array}{ll}1 & 0 \\ 0 & 0\end{array}\right), y=\left(\begin{array}{ll}0 & 0 \\ 0 & 1\end{array}\right), z=\left(\begin{array}{ll}1 & 1 \\ 1 & 1\end{array}\right) \in X$. Then we compute that

$$
\begin{aligned}
& o_{\ell}(x)=\left\{\left(\begin{array}{ll}
1 & 0 \\
0 & 0
\end{array}\right),\left(\begin{array}{ll}
0 & 0 \\
1 & 0
\end{array}\right),\left(\begin{array}{ll}
1 & 0 \\
1 & 0
\end{array}\right)\right\}=o_{r}(x)^{t}, \\
& o_{\ell}(y)=\left\{\left(\begin{array}{ll}
0 & 0 \\
0 & 1
\end{array}\right),\left(\begin{array}{ll}
0 & 1 \\
0 & 0
\end{array}\right),\left(\begin{array}{ll}
0 & 1 \\
0 & 1
\end{array}\right)\right\}=o_{r}(y)^{t}, \\
& o_{\ell}(z)=\left\{\left(\begin{array}{ll}
1 & 1 \\
1 & 1
\end{array}\right),\left(\begin{array}{ll}
0 & 0 \\
1 & 1
\end{array}\right),\left(\begin{array}{ll}
1 & 1 \\
0 & 0
\end{array}\right)\right\}=o_{r}(z)^{t} .
\end{aligned}
$$

Thus there exist three orbits with the same cardinality 3 under the left (right) regular action of $G$ on $X$.

Theorem 2.5. Let $R$ be a semisimple Artinian ring such that $X \neq \emptyset$. Then The following statements are equivalent:

(1) $R \cong \oplus_{i=1}^{m} M_{n_{i}}\left(D_{i}\right)$, with $D_{i}$ infinite division rings of the same cardinalities or $R$ is isomorphic to the ring of $2 \times 2$ matrices over a finite field.

(2) $\left|o_{\ell}(x)\right|=\left|o_{\ell}(y)\right|$ for all $x, y \in X$ under the left regular action on $X$ by $G$.

Proof. $(1) \Rightarrow(2)$. Assume (1) holds. Note that if $D$ is an infinite division ring, then any orbit $o_{\ell}(a)$ in $M_{n}(D), n \geq 2$ has at least $\left|D^{*}\right|$ elements since the regular action is free. But any subset of $M_{n}(D)$ has cardinality $\left|D^{n^{2}}\right|=|D|=$ $\left|D^{*}\right|$ if $D$ is infinite, so all orbits in $M_{n}(D)$ have the same cardinality. Hence if $R \cong \oplus_{i=1}^{m} M_{n_{i}}\left(D_{i}\right)$, with $D_{i}$ infinite division rings of the same cardinalities, then $\left|o_{\ell}(x)\right|=\left|o_{\ell}(y)\right|=\left|D_{i}\right|$ for all $x, y \in X$ under the left regular action on $X$ by $G$. If $R$ is isomorphic to the ring of $2 \times 2$ matrices over a field field $F$, then $\left|o_{\ell}(a)\right|=|F|^{2}-1$ for any $a \in X$ by Corollary 2.4 .

$(2) \Rightarrow(1)$. Assume (2) holds. By the Wedderburn-Artin Structure Theorem for a semisimple left Artinian ring, $R \cong \oplus_{i=1}^{m} M_{n_{i}}\left(D_{i}\right)$, where $M_{n_{i}}\left(D_{i}\right)$ is the full matrix ring of all $n_{i} \times n_{i}$ matrices over a division ring $D_{i}$ for each $i=$ $1,2, \ldots, m$ and for some positive integer $m$. Without loss of generality, we let $R=\oplus_{i=1}^{m} M_{n_{i}}\left(D_{i}\right)$. Consider the following two cases:

Case 1. All $D_{i}$ are infinite.

Assume that $\left|D_{i}\right| \neq\left|D_{j}\right|$ for some $i, j(i \neq j)$. Take $x=(0, \ldots, 0, a, 0, \ldots, 0)$, $y=(0, \ldots, 0, b, 0, \ldots, 0) \in X(R)$ where $a=\operatorname{diag}(1,0, \ldots, 0) \in M_{n_{i}}\left(D_{i}\right)$ and $b=\operatorname{diag}(1,0, \ldots, 0) \in M_{n_{j}}\left(D_{j}\right)$. Since $\left|D_{i}\right| \neq\left|D_{j}\right|$, clearly $\left|o_{\ell}(x)\right| \neq\left|o_{\ell}(y)\right|$, a contradiction. Hence $R=\oplus_{i=1}^{m} M_{n_{i}}\left(D_{i}\right)$, with $D_{i}$ infinite division rings of the same cardinalities.

Case 2. Some $D_{i}$ is finite.

Assume that there exists an infinite division ring $D_{j}$ for some $j(\neq i)$. Consider $x, y \in X(R)$ given in Case 1. Since $\left|D_{i}\right| \neq\left|D_{j}\right|$, clearly $\left|o_{\ell}(x)\right| \neq\left|o_{\ell}(y)\right|$, a contradiction. Hence all $D_{i}$ are finite division rings, and which are of the 
same cardinalities by the similar argument given in Case 1 . Let $F=D_{i}$ be a finite field for all $i$. Next assume that $m \geq 2$. Take $p=\left(1_{1}, 0, \ldots, 0\right)$, $q=\left(1_{1}, 1_{2}, \ldots, 0\right) \in X(R)$, where $1_{1}$ (resp. $\left.1_{2}\right)$ is the identity matrix of $M_{n_{1}}(F)$ (resp. $\left.M_{n_{2}}(F)\right)$. Clearly, $\left|o_{\ell}(p)\right| \neq\left|o_{\ell}(q)\right|$, a contradiction. Hence $m=1$, and so $R=M_{n_{1}}(F)$. Finally assume that $n_{1} \geq 3$. Take $\alpha=\operatorname{diag}(1,0, \ldots, 0), \beta=$ $(1,1,0, \ldots, 0) \in X(R)$. Then $\left|o_{\ell}(\alpha)\right| \neq\left|o_{\ell}(\beta)\right|$, also a contradiction. Hence $n_{1}=2$, and so $R$ is isomorphic to the ring of $2 \times 2$ matrices over a finite field.

\section{Rings with a finite number of orbits}

In [1], it was shown that if $R$ is a compact ring with identity and $X$ is a finite union of orbits under the regular or conjugation action, then $R$ is a finite ring. But in general, the result does not hold for some rings. For an example, if we consider an infinite division ring $D$, then in a ring $R=D \times D$ there exist only just two distinct orbits $o_{\ell}((1,0))\left(=o_{r}((1,0))\right)$ and $o_{\ell}((0,1))\left(=o_{r}((0,1))\right)$ under the left (right) regular action on $X$ by $G$ but $R$ is not a finite ring. In [2, Lemma 2.1], it was also shown that if $R$ is a ring with identity such that $X$ is a union of $n$ orbits under the regular action, then $x^{n+1}=0$ for all $x \in J$. This lemma could be improved as follows:

Lemma 3.1. Let $R$ be a ring such that $X \neq \emptyset$. If there exists a finite number of orbits under the left (resp. right) regular action of $G$ on $X$, then $R$ is a left Artinian ring (resp. right Artinian) ring. Moreover, $J^{n+1}=0$, where $n$ is the number of orbits under the left (resp. right) regular action of $G$ on $X$.

Proof. Assume that $R$ is not a left Artinian ring (resp. right Artinian) ring. Then there exists a strictly decreasing chain $\left\{I_{i}\right\}$ of infinite number of left (resp. right) ideals $I_{i}$ of $R$. Choose $x_{i} \in I_{i} \backslash I_{i+1}$ for each $i=1,2, \ldots$ Consider $o_{\ell}\left(x_{1}\right), o_{\ell}\left(x_{2}\right), \ldots$ (resp. $\left.o_{r}\left(x_{1}\right), o_{r}\left(x_{2}\right), \ldots\right)$. Then clearly these orbits under the left (resp. right) regular action of $G$ on $X$ are all distinct, a contradiction. Hence $R$ is a left Artinian ring (resp. right Artinian) ring. It is easy to show $J^{n+1}=0$ if there exist $n$ orbits under the left (resp. right) regular action of $G$ on $X$.

The following lemma was proved in [1, Lemma 2.2]. But we will have another proof of it.

Lemma 3.2. Let $R$ be a ring such that $X \neq \emptyset$. Then $G$ is transitive on $X$ under the left (resp. right) regular action on $X$ by $G$ if and only if $R$ is a local ring such that $J \neq 0=J^{2}$ and $J$ is a one-dimensional left (right) vector space over $R / J$.

Proof. Suppose that $G$ is transitive on $X$ under the regular left action on $X$ by $G$. First, we will show that $J \neq 0$. Indeed, if $J=(0)$, then by the WedderburnArtin Structure Theorem for a left Artinian ring $R, R \cong \oplus_{i=1}^{m} M_{n_{i}}\left(D_{i}\right)$, where $M_{n_{i}}\left(D_{i}\right)$ is the full matrix ring of all $n_{i} \times n_{i}$ matrices over a division ring 
$D_{i}$ for each $i=1,2, \ldots, m$ and for some positive integer $m$. Without loss of generality, we can let $R=\oplus_{i=1}^{m} M_{n_{i}}\left(D_{i}\right)$. If $n_{i} \geq 2$ for some $i$, then there are at least $1+\left|D_{i}\right| \geq 3$ orbits in $M_{n_{i}}\left(D_{i}\right)$ under the regular left action on $X\left(M_{n_{i}}\left(D_{i}\right)\right)$ by $G\left(M_{n_{i}}\left(D_{i}\right)\right)$ by Remark 1 , and also there are also at least $1+\left|D_{i}\right| \geq 3$ orbits in $R$ under the regular left action on $X$ by $G$, a contradiction. Hence $n_{i}=1$ for all $i$, i.e., $R \cong \oplus_{i=1}^{m} D_{i}$. If $m=1$, then $X=\emptyset$, a contradiction. Thus $R \cong \oplus_{i=1}^{m} D_{i}(m \geq 2)$, in which there exists at least two distinct orbits, which is also a contradiction. Therefore, $J \neq 0$. By Lemma $3.1, J^{2}=0$. Since $G$ is transitive on $X$ and $J \neq 0$, we have $J=X \cup\{0\}$, and so $R$ is a local ring. Indeed, if $g \notin J$ is not invertible, then for every $0 \neq x \in J, o_{\ell}(g)=o_{\ell}(x)$ which implies that $g=a x \in J$, a contradiction. Finally, since $R$ is local and $J^{2}=0 \neq J$, the map $\phi: R / J \times J \rightarrow J($ or $\psi: J \times R / J \rightarrow J)$ given by $\phi(a+J, b)=a b$ (or $\psi(b, a+J)=b a$ for all $a+J \in R / J, b \in J$ is well-defined. It is clear that $J$ is one-dimensional left vector space over $R / J$ with a basis $\{x\}$ for some $x \in X$. The proof of the converse is obvious. By the similar argument, we have the same result under the right regular action on $X$ by $G$.

Theorem 3.3. Let $R$ be a ring such that $X \neq \emptyset$ and the left (resp. right) regular action on $X$ by $G$ has exactly two orbits. Then $R$ is a local ring, or $R$ is isomorphic to a direct product of two division rings.

Proof. By Lemma 3.1, $J^{3}=0$. Let $X=o_{\ell}(x) \cup o_{\ell}(y)$ for some $x, y \in X$ under the left regular action on $X$ by $G$. Suppose that $J \neq(0)$. To show that $R$ is local, it is enough to show that $x, y \in J$. To get a contradiction, we may assume that $x \notin J, y \in J$. This means that $o_{\ell}(\bar{x})=\bar{X}$ in $\bar{R}=R / J$; by Lemma 3.2 this implies that $R / J$ is local, so $R$ is local. Next, suppose that $J=0$. Then by the Wedderburn-Artin Structure Theorem for left Artinian ring, $R \cong \oplus_{i=1}^{m} M_{n_{i}}\left(D_{i}\right)$, where $M_{n_{i}}\left(D_{i}\right)$ is the full matrix ring of all $n_{i} \times n_{i}$ matrices over a division ring $D_{i}$ for each $i=1,2, \ldots, m$ and for some positive integer $m$. Without loss of generality, we can let $R=\oplus_{i=1}^{m} M_{n_{i}}\left(D_{i}\right)$. If $n_{i} \geq 2$ for some $i$, then there exist at least $1+\left|D_{i}\right| \geq 3$ orbits in $R$ under the left regular action on $X$ by $G$ by Remark 1, a contradiction. Thus $R=\oplus_{i=1}^{m} D_{i}$. If $m \geq 3$, then there are at least three distinct orbits in $R$, which is also a contradiction. Hence $m=2$, and so $R$ is isomorphic to a direct product of two division rings. By the similar argument, we have the same result under the right regular action on $X$ by $G$.

Theorem 3.4. Let $R$ be a semisimple ring such that $X \neq \emptyset$. Then there are three orbits under the left (resp. right) regular action on $X$ by $G$ if and only if $R$ is isomorphic to $M_{2}\left(\mathbb{Z}_{2}\right)$, the ring of $2 \times 2$ matrices over $\mathbb{Z}_{2}$.

Proof. Suppose that there are three orbits under the left regular action on $X$ by $G$. By the Wedderburn-Artin Structure Theorem for left Artinian ring, $R \cong \oplus_{i=1}^{m} M_{n_{i}}\left(D_{i}\right)$, where $M_{i}\left(D_{i}\right)$ is the ring of $n_{i} \times n_{i}$ matrices over a division ring $D_{i}$ for each $i=1,2, \ldots, m$ and for some positive integer $m$. Without loss of generality, we can let $R=\oplus_{i=1}^{m} M_{n_{i}}\left(D_{i}\right)$. Assume that $R$ is commutative. 
Then $R=\oplus_{i=1}^{m} F_{i}$, where each $F_{i}$ is a field. In such a ring $R$, the number of orbits is $2^{m}-2$, and so three are not three orbits under the left regular action on $X$ by $G$, a contradiction. Hence $n_{i} \geq 2$ for some $i$. Since there are at least three distinct orbits under the left regular action on $X\left(M_{i}\left(D_{i}\right)\right)$ by $G\left(M_{i}\left(D_{i}\right)\right)$ by Remark 1, there are at least three distinct orbits under the left regular action on $X$ by $G$, and then $m=1$. Therefore, $R=M_{1}\left(D_{1}\right)$. If $n_{1} \geq 3$, then there are at least 4 nonzero singular matrices of $R$ which are not row-equivalent each other, and so there are at least 4 orbits under the left regular action on $X$ by $G$ by Lemma 2.1 , a contradiction. Hence $n_{1}=2$. If $\left|D_{1}\right| \geq 3$, then there are $\left|D_{1}\right|+1(\geq 4)$ orbits under the left regular action on $X$ by $G$ by Remark 1 , also a contradiction. Hence $\left|D_{1}\right|=2$, and so $D_{1} \simeq \mathbb{Z}_{2}$. Therefore, $R$ is isomorphic to $M_{2}\left(\mathbb{Z}_{2}\right)$. The converse is true by Example 1 . By the similar argument, we have the same result under the right regular action on $X$ by $G$.

Lemma 3.5. Let $R$ be a ring such that $X$ is a union of $n$ orbits under the left (resp. right) regular action on $X$ by $G$. If $J^{r} \neq 0$, then there are at most $(n-r)$ orbits under the left (resp right) regular action on $X(R / J)$ by $G(R / J)$.

Proof. Let $X=o_{\ell}\left(x_{1}\right) \cup o_{\ell}\left(x_{2}\right) \cup \cdots \cup o_{\ell}\left(x_{n}\right)$ for some $x_{1}, x_{1}, \ldots, x_{n} \in X$ under the left regular action on $X$ by $G$. By Lemma $3.1, J^{n+1}=0$. Since $J^{r} \neq 0=J^{n+1}$, we can choose $a_{i} \in J^{i} \backslash J^{i+1}$ for each $i=1,2, \ldots, r$. Clearly, $o_{\ell}\left(a_{1}\right), o_{\ell}\left(a_{2}\right), \ldots, o_{\ell}\left(a_{r}\right)$ are distinct. Hence without loss of generality we can let $o_{\ell}\left(a_{i}\right)=o_{\ell}\left(x_{i}\right)$ for each $i=1,2, \ldots, r$. Then $X(R / J)$ is a union of $(n-r)$ orbits $o_{\ell}\left(\overline{x_{r+1}}\right), \ldots, o_{\ell}\left(\overline{x_{n}}\right)$, where $\overline{x_{i}}=x_{i}+J \in X(R / J)$ for each $i=r+1, \ldots, n$ by $[4$, Proposition 4.8$]$. On the other hand, since these orbits are not necessarily distinct, there are at most $(n-r)$ orbits under the left regular action on $X(R / J)$ by $G(R / J)$. Similarly, the result holds under the right regular action on $X$ by G.

Corollary 3.6. Let $R$ be a ring such that $X$ is a union of $n(\geq 4)$ orbits under the left (resp. right) regular action on $X$ by $G$. Then we have the following:

(1) If $J^{n-1} \neq 0$, then $R$ is a local ring.

(2) If $J^{n-2} \neq 0$, then $R$ is a local ring or $R / J$ is isomorphic to a direct product of two division rings.

(3) If $J^{n-3} \neq 0$, then $R$ is a local ring or $R / J$ is isomorphic to a direct product of two division rings or $R / J$ is isomorphic to $M_{2}\left(\mathbb{Z}_{2}\right)$.

Proof. Let $\bar{R}=R / J, \bar{X}=X(\bar{R})$ and $\bar{G}=G(\bar{R})$.

(1) If $J^{n-1} \neq 0$, then there is at most one orbit under the left regular action on $\bar{X}$ by $\bar{G}$ by Lemma 3.5 , i.e., $\bar{X}=\emptyset$ or the left regular action on $\bar{X}$ by $\bar{G}$ is transitive. If $\bar{X}=\emptyset$, then $\bar{R}$ is a division ring, and so $R$ is a local ring. If the left regular action on $\bar{X}$ by $\bar{G}$ is transitive, then $\bar{R}$ is a local ring by Lemma 3.2 , and thus $R$ is a local ring.

(2) If $J^{n-2} \neq 0$, then there is at most two orbits under the left regular action on $\bar{X}$ by $\bar{G}$ by Lemma 3.5. Hence it follows from Lemma 3.2 and Theorem 3.3. 
(3) If $J^{n-3} \neq 0$, then there are at most three orbits under the left regular action on $\bar{X}$ by $\bar{G}$ by Lemma 3.5. If there is just one orbit, then $R / J$ is local by Lemma 3.2 , and thus $R$ is local. If there are two orbits, then $R / J$ is local or $R / J$ is isomorphic to a direct product of two division rings by Theorem 3.3. Finally, if there are three orbits of $\bar{G}$ on $\bar{X}$, then since $\bar{R}$ is semisimple, by Theorem 3.4 we get $R / J \cong M_{2}\left(\mathbb{Z}_{2}\right)$.

Similarly, the results also hold under the right regular action on $X$ by $G$.

Theorem 3.7. Let $R$ be a left Artinian ring. If $G$ is a cyclic group, then $R$ is a finite ring.

Proof. First, note that since $G$ is cyclic, $R / J=\prod_{i=1}^{m} F_{i}$, where $F_{i}$ is a field for each $i=1, \ldots, m$ and for some positive integer $m$. Also note that since each $F_{i}^{*}=F_{i} \backslash\{0\}$ is cyclic, and thus is finite by [5, page 426, Exercise 12]. Since $R$ is left Artinian, there is a composition series of left $R$-modules of finite length, $R=M_{0} \supset M_{1} \supset M_{2} \supset \cdots \supset M_{n-1} \supset M_{n}=(0)$. Since each factor $M_{i} / M_{i+1}$ $(i=0, \ldots, n-1)$ is simple $R$-module. Therefore, each $M_{i} / M_{i+1}$ is a direct summand of $R / J$, and so is finite. Thus $R$ is finite.

Corollary 3.8. Let $R$ be a ring such that $X$ is a finite union of orbits under the left (right) regular action on $X$ by $G$. If $G$ is a cyclic group, then $R$ is finite.

Proof. It follows from Lemma 3.1 and Theorem 3.7.

Acknowledgements. The authors would like to thank the referee for his/her careful checking of the details and helpful comments for making the paper more readable. In particular, Theorem 3.7 was proved quite simply by a nice suggestion of the referee.

\title{
References
}

[1] J. Cohen and K. Koh, Half-transitive group actions in a compact ring, J. Pure Appl. Algebra 60 (1989), no. 2, 139-153.

[2] J. Han, Regular action in a ring with a finite number of orbits, Comm. Algebra 25 (1997), no. 7, 2227-2236.

[3] Y. Hirano, Rings with finitely many orbits under the regular action, Rings, modules, algebras, and abelian groups, 343-347, Lecture Notes in Pure and Appl. Math., 236, Dekker, New York, 2004.

[4] T. Y. Lam, A First Course in Noncommutative Rings, Springer-Verlag, New York, 1991.

[5] W. K. Nicholson, Introduction to Abstract Algebra, PWS, Boston, 1998.

\author{
JUNCHEOL HAN \\ Department of Mathematics Education \\ Pusan National University \\ Pusan 609-735, Korea \\ E-mail address: jchan@pusan.ac.kr
}


RINGS WITH A FINITE NUMBER OF ORBITS UNDER THE REGULAR ACTION 663

\section{SANGWON PARK}

Department of Mathematics

Dong-A University

PUSAn 604-714, Korea

E-mail address: swpark@donga.ac.kr 